\title{
Comparative Solution of Nonlinear Quintic Cubic Oscillator Using Modified Homotopy Perturbation Method
}

\author{
Muhammad Suleman ${ }^{1,2}$ and Qingbiao $\mathrm{Wu}^{1}$ \\ ${ }^{1}$ Department of Mathematics, Zhejiang University, Hangzhou, China \\ ${ }^{2}$ Department of Mathematics, Comsat Institute of Information Technology, Islamabad, Pakistan \\ Correspondence should be addressed to Muhammad Suleman; suleman@zju.edu.cn
}

Received 1 April 2015; Revised 14 May 2015; Accepted 17 May 2015

Academic Editor: Nikos Mastorakis

Copyright ( 2015 M. Suleman and Q. Wu. This is an open access article distributed under the Creative Commons Attribution License, which permits unrestricted use, distribution, and reproduction in any medium, provided the original work is properly cited.

\begin{abstract}
We use modified homotopy perturbation method to find out the solution of nonlinear cubic quintic equation. Besides this method solution of the problem with the following methods is discussed, Energy Balance Method and He's Frequency Formulation method and then compare the results with each other and Global Error Method. The results show that these three methods are effective as global error method for nonlinear cubic quintic oscillator equation with multiple nonlinear terms and have different effects on the solution. In particular, the homotopy perturbation solution is quite surprising. A cubic quintic nonlinear oscillator is used as an example to compare the results.
\end{abstract}

\section{Introduction}

In the present era large numbers of engineering problems are nonlinear and it is almost difficult to solve them analytically. Perturbation methods are among the known methods to solve the nonlinear problems, which are based on the existence of small/large parameters so-called perturbation parameters. These so-called perturbation parameters are not present in many nonlinear problems so we cannot use perturbation methods. To overcome this difficulty researchers introduced such methods which do not require small parameters such as homotopy perturbation method (HPM) [1, 2], book keeping parameter perturbation method [3], He's energy balance method (EBM) [4-7], global error minimization method [8], Variational Iterational method [4], and many others. In this paper, solution of nonlinear cubic quintic oscillatory equation is solved with iterative methods, the basic idea of the homotopy perturbation is used, and its applications in some nonlinear oscillator equations are studied. In most engineering problems, it is easy to find angular frequency of a solution to a nonlinear equation. He presented the method for the first time. These results are valid for weakly nonlinear systems as well as strongly nonlinear systems. In Section 2, solution with energy balance method is explained. Solution with He's frequency formulation method is explained in Section 3. In Section 4, HPM solution is explained and some conclusions are given in Section 5.

\section{Energy Balance Method (EBM)}

Consider the nonlinear quintic cubic equation

$$
\phi^{\prime \prime}+\phi+\varepsilon_{1} \phi^{3}+\varepsilon_{2} \phi^{5}=0 \quad \phi(0)=A, \phi^{\prime}(0)=0 .
$$

Its variational principle can be easily obtained:

$$
J(\phi)=\int_{0}^{t}\left(-\frac{1}{2} \phi^{\prime 2}+\frac{1}{2} \phi^{2}+\frac{1}{4} \varepsilon_{1} \phi^{4}+\frac{1}{6} \varepsilon_{2} \phi^{6}\right) d t .
$$

Its Hamiltonian, therefore, can be written in the form

$$
\begin{aligned}
H(\phi)= & \frac{1}{2} \phi^{\prime 2}+\frac{1}{2} \phi^{2}+\frac{1}{4} \varepsilon_{1} \phi^{4}+\frac{1}{6} \varepsilon_{2} \phi^{6} \\
= & \frac{1}{2} A^{2}+\frac{1}{4} \varepsilon_{1} A^{4}+\frac{1}{6} \varepsilon_{2} A^{6}, \\
H= & \frac{1}{2} \phi^{\prime 2}+\frac{1}{2} \phi^{2}+\frac{1}{4} \varepsilon_{1} \phi^{4}+\frac{1}{6} \varepsilon_{2} \phi^{6}-\frac{1}{2} A^{2} \\
& -\frac{1}{4} \varepsilon_{1} A^{4}-\frac{1}{6} \varepsilon_{2} A^{6}=0 .
\end{aligned}
$$


In (3) and (4), the kinetic energy $(E)$ and potential energy (T) can be, respectively, expressed as $\left((1 / 2) \phi^{\prime 2},(1 / 2) \phi^{2}+\right.$ $\left.(1 / 4) \varepsilon_{1} \phi^{4}+(1 / 6) \varepsilon_{2} \phi^{6}\right)$ throughout the oscillation; it holds that $H=E+T=$ constant. We use the following trial function to determine the angular frequency $\omega$. Let

$$
\phi=A \cos t \omega t .
$$

Being the trial function, substituting (5) into (4), we obtain the following residual equation:

$$
\begin{aligned}
& R(t)=\frac{1}{2} A^{2} \omega^{2} \sin ^{2} \omega t+\frac{1}{2} A^{2} \cos ^{2} \omega t+\frac{1}{4} \varepsilon_{1} A^{4} \cos ^{4} \omega t \\
& \quad+\frac{1}{6} \varepsilon_{2} A^{6} \cos ^{6} \omega t-\frac{1}{2} A^{2}-\frac{1}{4} \varepsilon_{1} A^{4}-\frac{1}{6} \varepsilon_{2} A^{6} \\
& =\frac{1}{2} A^{2}\left[\omega^{2} \sin ^{2} \omega t+\cos ^{2} \omega t+\frac{1}{2} \varepsilon_{1} A^{2} \cos ^{4} \omega t\right. \\
& \left.\quad+\frac{1}{3} \varepsilon_{2} A^{4} \cos ^{6} \omega t-1-\frac{1}{2} \varepsilon_{1} A^{2}-\frac{1}{3} \varepsilon_{2} A^{4}\right], \\
& R(t)=\omega^{2} \sin ^{2} \omega t+\cos ^{2} \omega t+\frac{1}{2} \varepsilon_{1} A^{2} \cos ^{4} \omega t+\frac{1}{3} \varepsilon_{2} A^{4} \\
& \quad \cdot \cos ^{6} \omega t-1-\frac{1}{2} \varepsilon_{1} A^{2}-\frac{1}{3} \varepsilon_{2} A^{4} .
\end{aligned}
$$

If, by chance, the exact solution had been chosen as the trial function, then it would be possible to make residual $R(t)$ zero for all values of " $t$ " by appropriate choice of $\omega$. Since (5) is only an approximation to the exact solution, $R(t)$ cannot be made zero everywhere.

Collocation at $\omega t=\pi / 4$ gives

$$
\omega=\sqrt{1+\frac{3}{4} \varepsilon_{1} A^{2}+\frac{5}{12} \varepsilon_{2} A^{4} .}
$$

\section{He's Frequency Formulation Method}

Equation (1) can be written in the general form

$$
\phi^{\prime \prime}+f(\phi)=0 \quad \phi(0)=A, \phi^{\prime}(0)=0 .
$$

We use two trial functions $\phi_{1}(t)=A$ cost and $\phi_{2}(t)=$ $A \cos \omega t$, which are, respectively, the solutions of the following linear oscillator equations:

$$
\begin{array}{ll}
\phi^{\prime \prime}+\omega_{1}^{2} \phi=0, & \omega_{1}^{2}=1, \\
\phi^{\prime \prime}+\omega_{2}^{2} \phi=0, & \omega_{2}^{2}=\omega^{2},
\end{array}
$$

where $\omega$ is assumed to be the frequency of the nonlinear oscillator. The residuals for (8) are

$$
\begin{aligned}
R_{1}(t) & =-A \cos t+f(A \cos t), \\
R_{2}(\omega t) & =-A \omega^{2} \cos \omega t+f(A \cos \omega t) .
\end{aligned}
$$

The original frequency-amplitude formulation reads [9]

$$
\omega^{2}=\frac{\omega_{1}^{2} R_{2}-\omega_{2}^{2} R_{1}}{R_{2}-R_{1}} .
$$

We just used the following formulation:

$$
\omega^{2}=\frac{\omega_{1}^{2} R_{2}(0)-\omega_{2}^{2} R_{1}(0)}{R_{2}-R_{1}} .
$$

Geng and Cai improved the formulation by choosing another location point [10]:

$$
\omega^{2}=\frac{\omega_{1}^{2} R_{2}(\pi / 3)-\omega_{2}^{2} R_{1}(\pi / 3)}{R_{2}-R_{1}} .
$$

The accuracy depends upon the chosen location point, and we have no general rule for the choice.

To illustrate this shortcoming, we consider the cubic quintic nonlinear equation [8]:

$$
\phi^{\prime \prime}+\phi+\varepsilon_{1} \phi^{3}+\varepsilon_{2} \phi^{5}=0, \quad \phi(0)=A, \phi^{\prime}(0)=0 .
$$

The residual of the equations is

$$
\begin{aligned}
R_{1}(t)= & \varepsilon_{1} A^{3} \cos ^{3} t+\varepsilon_{2} A^{5} \cos ^{5} t \\
R_{2}(\omega t)= & \left(1-\omega^{2}\right) A \cos \omega t+\varepsilon_{1} A^{3} \cos ^{3} \omega t \\
& +\varepsilon_{2} A^{5} \cos ^{5} \omega t
\end{aligned}
$$

where frequency can be calculated at 0 or $\pi / 3$ :

$$
\begin{aligned}
& \omega^{2}=\frac{\omega_{1}^{2} R_{2}(0)-\omega_{2}^{2} R_{1}(0)}{R_{2}-R_{1}}, \\
& \omega^{2}=\frac{\omega_{1}^{2} R_{2}(\pi / 3)-\omega_{2}^{2} R_{1}(\pi / 3)}{R_{2}-R_{1}} .
\end{aligned}
$$

Now locating $\cos t=\cos \omega t=k$ (where $0<k<1, k=1$, for (15) and $k=1 / 2$ for (16)), we obtain

$$
\omega^{2}=1+\varepsilon_{1} A^{2} k^{2}+\varepsilon_{2} A^{4} k^{4}, \quad 0<k<1 .
$$

It shows that the accuracy depends on the point of location. 
3.1. The Method of Weighted Residual. We use the method of weighted residuals to overcome the shortcoming. To this end, we introduce two new residual variables $\widetilde{R}_{1}$ and $\widetilde{R}_{2}$ defined as

$$
\begin{aligned}
\widetilde{R}_{1} & =\frac{4}{T_{1}} \int_{0}^{T_{1} / 4} R_{1}(t) \cos \left(\frac{2 \pi}{T_{1}} t\right) d t, \quad T_{1}=2 \pi \\
= & \frac{4}{2 \pi} \int_{0}^{\pi / 2}\left(\varepsilon_{1} A^{3} \cos ^{3} t+\varepsilon_{2} A^{5} \cos ^{5} t\right) \cos t d t=\frac{2}{\pi} \\
& \cdot \int_{0}^{\pi / 2}\left(\varepsilon_{1} A^{3} \cos ^{4} t+\varepsilon_{2} A^{5} \cos ^{6} t\right) d t, \\
\widetilde{R}_{1}= & \frac{3}{8} \varepsilon_{1} A^{3}+\frac{5}{16} \varepsilon_{2} A^{5}, \\
\widetilde{R}_{2}= & \frac{4}{T_{2}} \int_{0}^{T_{1} / 4} R_{2}(t) \cos \left(\frac{2 \pi}{T_{2}} t\right) d t, \quad T_{2}=\frac{2 \pi}{\omega} \\
= & \frac{4}{2 \pi / \omega} \int_{0}^{\pi / 2 \omega}\left(\left(1-\omega^{2}\right) A \cos \omega t+\varepsilon_{1} A^{3} \cos ^{3} \omega t\right. \\
& \left.+\varepsilon_{2} A^{5} \cos ^{5} \omega t\right) \cos \omega t d t=\frac{2}{\pi} \int_{0}^{\pi / 2}\left(1-\omega^{2}\right) A \cos ^{2} r \\
& +\varepsilon_{1} A^{3} \cos ^{4} r+\varepsilon_{2} A^{5} \cos ^{6} r d r, \\
\widetilde{R}_{2}= & A\left(1-\omega^{2}\right)+\frac{3}{8} \varepsilon_{1} A^{3}+\frac{5}{16} \varepsilon_{2} A^{5} .
\end{aligned}
$$

We can approximately calculate $\omega^{2}$ in the form

$$
\omega^{2}=\frac{\omega_{1}^{2} \widetilde{R}_{2}-\omega_{2}^{2} \widetilde{R}_{1}}{\widetilde{R}_{2}-\widetilde{R}_{1}} .
$$

Now, by using (20) for frequency formulation, we find the value of $\omega^{2}$ for cubic quintic nonlinear oscillator:

$$
\omega^{2}=1+\frac{3}{4} \varepsilon_{1} A^{2}+\frac{5}{8} \varepsilon_{2} A^{4} .
$$

Its approximate frequency reads

$$
\omega=\sqrt{1+\frac{3}{4} \varepsilon_{1} A^{2}+\frac{5}{8} \varepsilon_{2} A^{4}} .
$$

\section{Modified Homotopy Perturbation Method with Two Expanding Parameters}

Consider the following nonlinear cubic quintic oscillator [8]:

$$
\phi^{\prime \prime}+\phi+\varepsilon_{1} \phi^{3}+\varepsilon_{2} \phi^{5}=0, \quad \phi(0)=A, \phi^{\prime}(0)=0,
$$

where $\varepsilon_{1}$ and $\varepsilon_{2}$ are constant. Equation (23) is exactly solvable and its solution is given in [11]. According to homotopy perturbation method $[1,2]$, homotopy equation can be constructed in the form

$$
\phi^{\prime \prime}+\omega^{2} \phi+p\left[\varepsilon_{1} \phi^{3}+\varepsilon_{2} \phi^{5}+\left(1-\omega^{2}\right) \phi\right]=0,
$$

where $\omega$ is unknown frequency of oscillator to be determined. When $p=0$, we have

$$
\phi^{\prime \prime}+\omega^{2} \phi=0 .
$$

This can be approximately describing the solution properties. Embedding parameter $p$ increases monotonically from zero to unit as the trivial problem. Equation (25) is continuously deformed to the original one. According to standard homotopy perturbation method $[1,2]$, solution is expanded in a series of $p$ :

$$
\phi=\phi_{0}+p \phi_{1}+p^{2} \phi_{2}+p^{3} \phi_{3}+\cdots
$$

Generally one iteration is enough to obtain an approximate solution with relatively high accuracy. If higher accuracy of solution is needed, homotopy equation is suggested in the following [1]:

$$
\phi^{\prime \prime}+1 \cdot \phi+p\left[\varepsilon_{1} \phi^{3}+\varepsilon_{2} \phi^{5}\right]=0
$$

Solution is also expanded in the same form of (26) and coefficient 1 of the linear term in (27) is also expanded in series of $p$ :

$$
1=\omega^{2}+p \omega_{1}+p^{2} \omega_{2}+p^{3} \omega_{3}+\cdots
$$

This method is also used in parameter-expansion method. In [6] a homotopy equation with an auxiliary term is suggested which vanishes for both cases with homotopy parameters $p=0$ and $p=1$ in constructed homotopy equation. A detailed solution process is given and a relatively comprehensive survey on concepts, theory, and applications of homotopy perturbation method is reported $[1-3,12]$. In this paper, we suggest a homotopy perturbation method with two expanding parameters.

4.1. Solution of Problem Using HPM. In (23) there are two nonlinear terms, which might have different effects on the solution. In order to find the best match with different nonlinear terms, we can construct a homotopy equation in the form

$$
\phi^{\prime \prime}+1 \cdot \phi+p_{1} \varepsilon_{1} \phi^{3}+p_{2} \varepsilon_{2} \phi^{5}=0,
$$

where $p_{1}$ and $p_{2}$ are homotopy parameters, $p_{1} \in[0,1]$, $p_{2} \in[0,1]$. Solution and coefficient 1 of linear term in (29) are expanded in the form

$$
\begin{aligned}
\phi= & \phi_{0}+p_{1} \phi_{1}+p_{2} \phi_{2}+p_{1}^{2} \phi_{3}+p_{1} p_{2} \phi_{4}+p_{2}^{2} \phi_{5}+\cdots, \\
1= & \omega^{2}+p_{1} \omega_{1}+p_{2} \omega_{2}+p_{1}^{2} \omega_{3}+p_{1} p_{2} \omega_{4}+p_{2}^{2} \omega_{5} \\
& +\cdots
\end{aligned}
$$

This expansion is similar to modified Lindstedt-Poincare method with double series expansion [3]. Substituting (30) and (31) into (29), collecting the same power of 
$p_{1}^{m} p_{2}^{n}(m, n=0,1,2,3, \ldots)$, and setting coefficients zero, we can readily obtain the following equations:

$$
\begin{array}{r}
\phi_{0}^{\prime \prime}+\omega^{2} \phi_{0}=0, \\
\phi(0)=A, \phi^{\prime}(0)=0, \\
\phi_{1}^{\prime \prime}+\omega^{2} \phi_{1}+\omega_{1} \phi_{0}+\varepsilon_{1} \phi_{0}^{3}=0, \\
\phi_{1}(0)=0, \phi_{1}^{\prime}(0)=0, \\
\phi_{2}^{\prime \prime}+\omega^{2} \phi_{2}+\omega_{2} \phi_{0}+\varepsilon_{2} \phi_{0}^{5}=0, \\
\phi_{2}(0)=0, \phi_{2}^{\prime}(0)=0, \\
\phi_{3}^{\prime \prime}+\omega^{2} \phi_{3}+\omega_{3} \phi_{0}+\omega_{1} \phi_{1}+3 \varepsilon_{1} \phi_{0}^{2} \phi_{1}=0, \\
\phi_{3}(0)=0, \phi_{3}^{\prime}(0)=0, \\
\phi_{4}^{\prime \prime}+\omega^{2} \phi_{4}+\omega_{4} \phi_{0}+\omega_{1} \phi_{2}+3 \varepsilon_{1} \phi_{0}^{2} \phi_{2}+5 \varepsilon_{2} \phi_{0}^{4} \phi_{1}^{5}=0, \\
\phi_{4}(0)=0, \phi_{4}^{\prime}(0)=0, \\
\phi_{5}^{\prime \prime}+\omega^{2} \phi_{5}+\omega_{5} \phi_{0}+\omega_{1} \phi_{2}+3 \varepsilon_{1} \phi_{0}^{2} \phi_{2}+5 \varepsilon_{2} \phi_{0}^{4} \phi_{2}^{5}=0, \\
\phi_{5}(0)=0, \phi_{5}^{\prime}(0)=0 .
\end{array}
$$

Initial conditions for $\phi_{i}(i>1)$ can be freely chosen, but it must hold that $\phi(0)=A$ and $\phi^{\prime}(0)=0$ for final closed approximate solution. Solving (32), we have

$$
\phi_{0}(t)=A \cos \omega t .
$$

Substitution of $\phi_{0}$ into (33) results in

$$
\begin{aligned}
\phi_{1}^{\prime \prime}+ & \omega^{2} \phi_{1}+A\left(\omega_{1}+\varepsilon_{1} A^{2} \frac{3}{4}\right) \cos \omega t \\
+ & \frac{1}{4} \varepsilon_{1} A^{3} \cos 3 \omega t=0 .
\end{aligned}
$$

Requiring no secular term in $\phi_{1}$ from (39), we have

$$
\omega_{1}=-\varepsilon_{1} A^{2} \frac{3}{4}
$$

A particular solution for (39) is readily obtained as

$$
\phi_{1}=\frac{1}{36 \omega^{2}} \varepsilon_{1} A^{3} \cos 3 \omega t .
$$

Substituting $\omega_{1}, \phi_{0}$, and $\phi_{1}$ into (34) one gets

$$
\begin{aligned}
\phi_{2}^{\prime \prime}+ & \omega^{2} \phi_{2}+A\left(\omega_{2}+\frac{3}{8} \varepsilon_{2} A^{4}\right) \cos \omega t \\
& +\frac{5}{16} \varepsilon_{2} A^{5} \cos 3 \omega t+\frac{1}{16} \varepsilon_{2} A^{5} \cos 5 \omega t=0 .
\end{aligned}
$$

In order to remove a secular term from (42), consider

$$
\omega_{2}=-\frac{3}{8} \varepsilon_{2} A^{4} .
$$

Now obtain a particular solution for (42):

$$
\phi_{2}=\frac{5}{144 \omega^{2}} \varepsilon_{2} A^{5} \cos 3 \omega t+\frac{1}{250 \omega^{2}} \varepsilon_{2} A^{5} \cos 5 \omega t .
$$

Substituting the obtained results into (35), we obtain equation for $\omega_{3}$ and $\phi_{3}$, respectively:

$$
\begin{aligned}
\phi_{3}^{\prime \prime} & +\omega^{2} \phi_{3}+\omega_{3} A \cos \omega t \\
& +\left(-\varepsilon_{1} A^{2} \frac{3}{4}\right)\left(\frac{1}{36 \omega^{2}} \varepsilon_{1} A^{3} \cos 3 \omega t\right) \\
& +3 \varepsilon_{1}(A \cos \omega t)^{2}\left(\frac{1}{36 \omega^{2}} \varepsilon_{1} A^{3} \cos 3 \omega t\right)=0, \\
\omega_{3} & =-\frac{1}{48} \varepsilon_{1} A^{4}, \\
\phi_{3} & =\frac{1}{25 \omega^{2}} \varepsilon_{1}^{2} A^{5} \cos 5 \omega t+\frac{1}{25 \omega^{2}} \varepsilon_{1}^{2} A^{5} .
\end{aligned}
$$

Similarly, we can find out the values of $\omega_{4}$ and $\omega_{5}$ by using (36) and (37):

$$
\begin{aligned}
& \omega_{4}=0, \\
& \omega_{5}=-\frac{35}{576 \omega^{2}} \varepsilon_{2}^{2} A^{8} .
\end{aligned}
$$

The solution process continues in this way. Substituting the values of $\omega_{i}(i=1-5)$ into (31) and using $p_{1}=1$ and $p_{2}=1$, we have

$$
\begin{aligned}
1 & =\omega^{2}-\frac{3}{4} \varepsilon_{1} A^{2}-\frac{3}{8} \varepsilon_{2} A^{4}-\frac{1}{24 \omega^{2}} \varepsilon_{1}^{2} A^{4}-\frac{35}{576 \omega^{2}} \varepsilon_{2}^{2} A^{8}, \\
\omega & =\sqrt{\frac{1+(3 / 4) \varepsilon_{1} A^{2}+\sqrt{\left(\left(1+(3 / 4) \varepsilon_{1} A^{2}\right)^{2}+(15 / 4) \varepsilon_{1}^{2} A^{4}+(35 / 144) \varepsilon_{2}^{2} A^{4}\right)}}{2}} .
\end{aligned}
$$

According to Nayfeh's result [13] for comparison

$$
\omega=1+\left(\frac{3}{8} \varepsilon_{2}-\frac{5}{12} \varepsilon_{1}^{2}\right) A^{2} .
$$

This result is obtained under the assumption of small amplitude. Consequently, the above methods give the same result as that of Nayfeh [13]. In case of $\varepsilon_{2}$ being zero it will become 
TABLE 1: Comparison of HPM, He’s frequency, energy balance method, global error method, and exact solution.

\begin{tabular}{|c|c|c|c|c|c|}
\hline$A$ (amplitude) & Modified HPM & Energy balance method & He's frequency & Global error minimization method & Exact \\
\hline 0.2 & 1.0724 & 1.0826 & 1.0998 & 1.0726 & 1.0720 \\
\hline 0.5 & 1.1954 & 1.4701 & 1.4764 & 1.2438 & 1.1846 \\
\hline 1 & 1.9947 & 1.9254 & 1.9635 & 2.3262 & 1.3778 \\
\hline 5 & 2.0017 & 2.5217 & 2.5321 & 2.1980 & 2.1504 \\
\hline 10 & 2.3434 & 2.7423 & 2.8984 & 2.9463 & 2.8666 \\
\hline 100 & 7.1033 & 8.1798 & 8.1975 & 8.8327 & 8.5336 \\
\hline
\end{tabular}

the well-known Duffing equation and its nonlinear angular frequency can be obtained. The result is the same as that obtained in [13] and its maximal relative error is less than $7.5 \%$.

\section{Conclusions}

From Table 1 it is suggested that these methods not only are effective for the given nonlinear cubic quintic oscillatory equation but also can be extended to various nonlinear problems with multiple nonlinear terms. In particular, the solution procedure of modified homotopy perturbation method with two expanding parameters can be used as a paradigm for many other applications.

\section{Conflict of Interests}

The authors declare that there is no conflict of interests regarding the publication of this paper.

\section{Acknowledgments}

This work is supported by the National Natural Science Foundation of China (Grant no. 11371320), Zhejiang Natural Science Foundation (Grant no. LZ4A010002), Foundation of Science and Technology Department (Grant no. 2013C31084) of Zhejiang province, and Scientific Research Fund of Zhejiang Provincial Education Department (Grants nos. Y201431077 and Y2013329420).

\section{References}

[1] J. H. He, "Homotopy perturbation method with two expanding parameters," Indian Journal of Physics, vol. 88, no. 2, pp. 193-196, 2014.

[2] J.-H. He, "Bookkeeping parameter in perturbation methods," International Journal of Nonlinear Sciences and Numerical Simulation, vol. 2, no. 3, pp. 257-264, 2001.

[3] H.-L. Zhang, "Periodic solutions for some strongly nonlinear oscillations by He's energy balance method," Computers \& Mathematics with Applications, vol. 58, no. 11-12, pp. 2480-2485, 2009.

[4] N. Jamshidi and D. D. Ganji, "Application of energy balance method and variational iteration method to an oscillation of a mass attached to a stretched elastic wire," Current Applied Physics, vol. 10, no. 2, pp. 484-486, 2010.

[5] S. S. Ganji, D. D. Ganji, Z. Z. Ganji, and S. Karimpour, "Periodic solution for strongly nonlinear vibration systems by He's energy balance method," Acta Applicandae Mathematicae, vol. 106, no. 1, pp. 79-92, 2009.

[6] J.-H. He, "Homotopy perturbation method with an auxiliary term," Abstract and Applied Analysis, vol. 2012, Article ID 857612, 7 pages, 2012.

[7] J.-H. He, "Comment on He's frequency formulation for nonlinear oscillators," European Journal of Physics, vol. 29, no. 4, 2008.

[8] A. Kargar and M. Akbarzade, "Analytical solution of nonlinear cubic-quintic Duffing oscillator using global error minimization method," Advanced Studies in Theoretical Physics, vol. 6, no. 9-12, pp. 467-471, 2012.

[9] M. Gorji, D. D. Ganji, and S. Soleimani, "New application of He's homotopy perturbation method," International Journal of Nonlinear Sciences and Numerical Simulation, vol. 8, no. 3, pp. 319-328, 2007.

[10] L. Geng and X.-C. Cai, "He's frequency formulation for nonlinear oscillators," European Journal of Physics, vol. 28, no. 5, pp. 923-931, 2007.

[11] A. Elías-Zúñiga, "Exact solution of the cubic-quintic Duffing oscillator," Applied Mathematical Modelling, vol. 37, no. 4, pp. 2574-2579, 2013.

[12] M. Febbo, "A finite extensibility nonlinear oscillator," Applied Mathematics and Computation, vol. 217, no. 14, pp. 6464-6475, 2011.

[13] A. H. Nayfeh, Introduction to Perturbation Technique, John Wiley \& Sons, New York, NY, USA, 1985. 


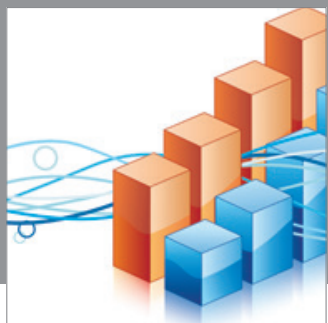

Advances in

Operations Research

mansans

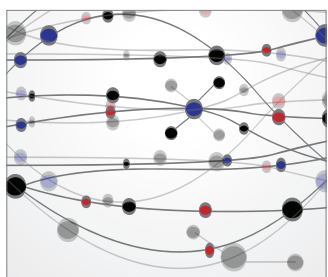

The Scientific World Journal
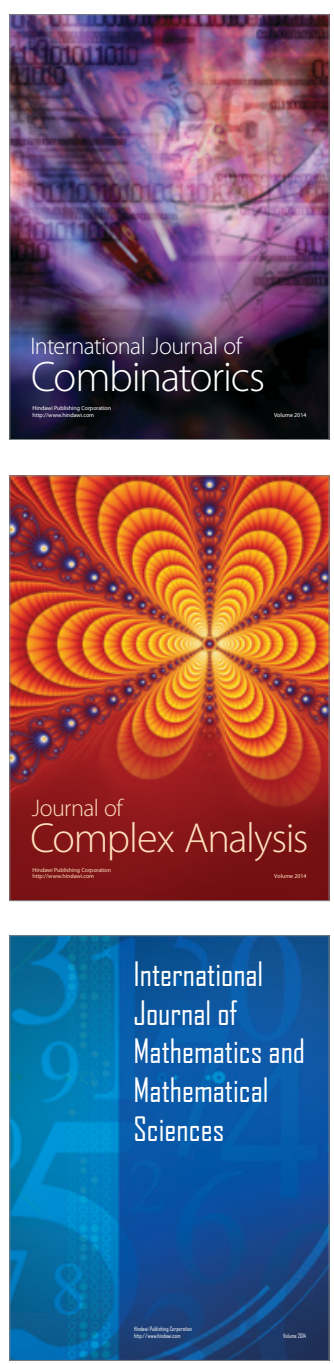
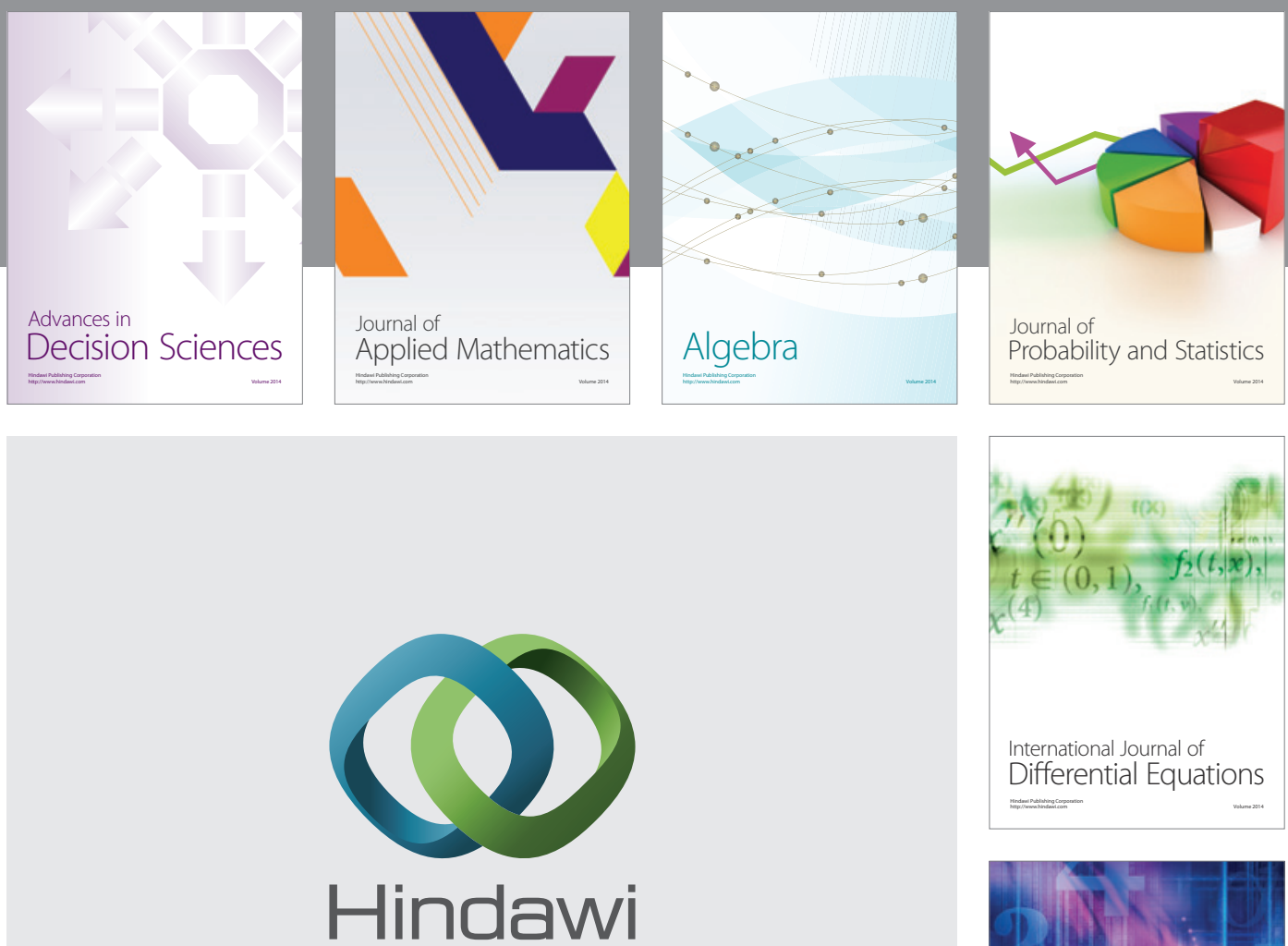

Submit your manuscripts at http://www.hindawi.com
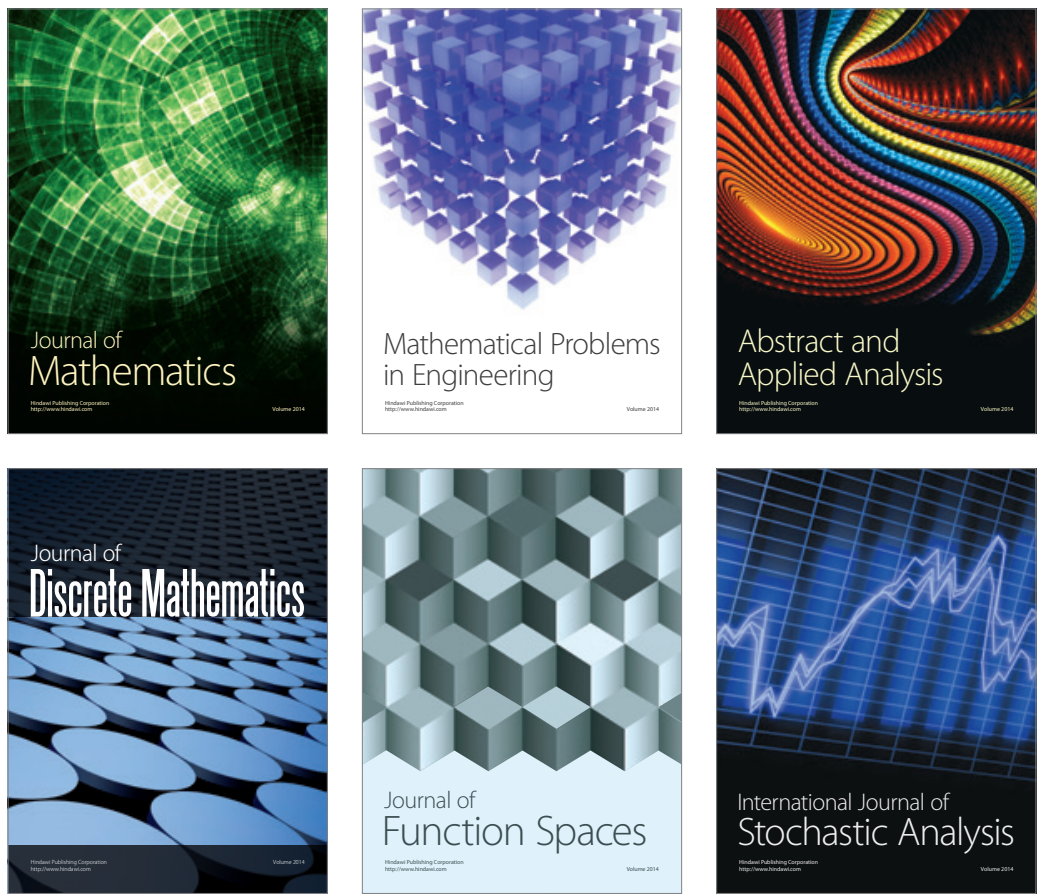

Journal of

Function Spaces

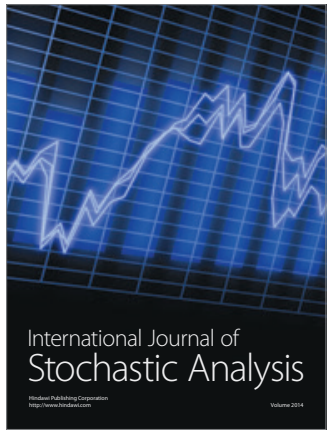

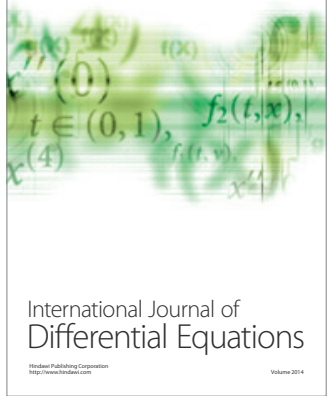
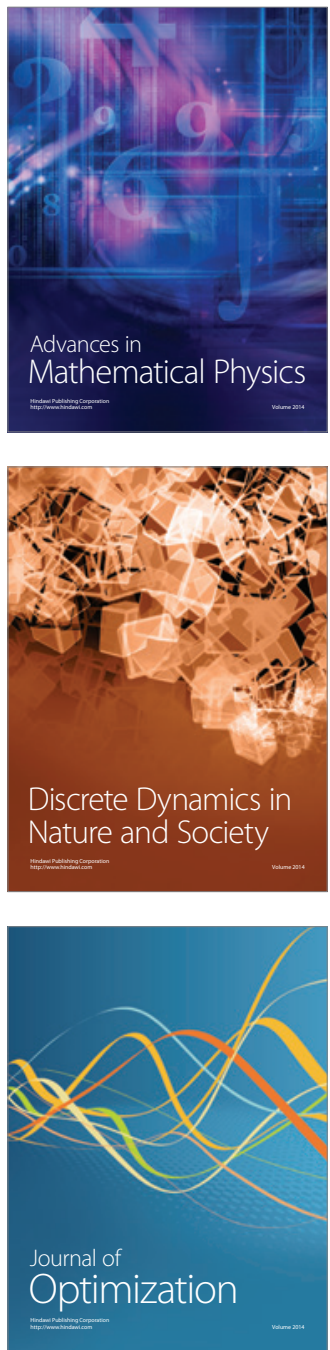